\title{
Incorporation of Interfacial Intermetallic Morphology in Fracture Mechanism Map for Sn-Ag-Cu Solder Joints
}

\author{
Z. HUANG,${ }^{1}$ P. KUMAR,${ }^{2}$ I. DUTTA,${ }^{1,4}$ R. SIDHU,${ }^{3}$ M. RENAVIKAR, ${ }^{3}$ \\ and R. MAHAJAN ${ }^{3}$ \\ 1.-School of Mechanical and Materials Engineering, Washington State University, Pullman, \\ WA 99164, USA. 2.-Department of Materials Engineering, Indian Institute of Science, Bangalore \\ 560012, India. 3.-Intel Corp., Assembly Technology Development, 5000 W. Chandler Blvd., \\ Chandler, AZ 85226, USA. 4.-e-mail: idutta@wsu.edu
}

A fracture mechanism map (FMM) is a powerful tool which correlates the fracture behavior of a material to its microstructural characteristics in an explicit and convenient way. In the FMM for solder joints, an effective thickness of the interfacial intermetallic compound (IMC) layer $\left(t_{\mathrm{eff}}\right)$ and the solder yield strength $\left(\sigma_{\mathrm{ys}, \text { eff }}\right)$ are used as abscissa and ordinate axes, respectively, as these two predominantly affect the fracture behavior of solder joints. Earlier, a definition of $t_{\text {eff }}$, based on the uniform thickness of IMC $\left(t_{\mathrm{u}}\right)$ and the average height of the IMC scallops $\left(t_{\mathrm{s}}\right)$, was proposed and shown to aptly explain the fracture behavior of solder joints on $\mathrm{Cu}$. This paper presents a more general definition of $t_{\text {eff }}$ that is more widely applicable to a range of metallizations, including $\mathrm{Cu}$ and electroless nickel immersion gold (ENIG).

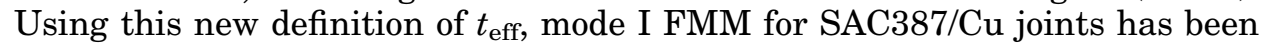
updated and its validity was confirmed. A preliminary FMM for SAC387/Cu joints with ENIG metallization is also presented.

Key words: Solder joint fracture, fracture mechanism map (FMM), effective thickness of IMC

\section{INTRODUCTION}

In microelectronic devices, low-cycle fatigue cracks form in solder joints due to thermalmechanical cycling during service. ${ }^{1-3}$ These preexisting cracks may propagate under a combination of tensile and shear loading, especially when the device sustains impact loading under drop conditions. Hence, the study of the fracture behavior of solder joints is vital for designing robust packages.

Several reports ${ }^{4-12}$ addressing the fracture behavior of solder joints are available in the literature. However, until recently, ${ }^{10-12}$ mixed-mode fracture of solder joints under high-strain-rate conditions, which is applicable to failure of electronic solders due to accidental drops, had not been studied. Recent work by the present authors reported on the development of fracture mechanism

(Received May 28, 2013; accepted August 19, 2013;

published online September 20, 2013) maps (FMMs) for $\mathrm{Sn}-\mathrm{Ag}-\mathrm{Cu}$ solder joints on $\mathrm{Cu}$ substrates, ${ }^{11}$ where the various operative fracture mechanisms are represented in a two-dimensional space described by an effective solder yield strength on the ordinate and an effective interfacial intermetallic compound (IMC) thickness on the abscissa. In this paper, we present a modification of the previously used definition of the effective IMC thickness, in order to make it more generally applicable to systems besides solder-on-Cu.

\section{BACKGROUND}

In previous work, ${ }^{10-12}$ we presented detailed analysis of the fracture behavior of $\mathrm{Sn}-3.8 \mathrm{Ag}-0.7 \mathrm{Cu}$ (SAC387) solder joints attached to $\mathrm{Cu}$ substrates under both quasistatic and dynamic loading conditions (strain range of $0.01 \mathrm{~s}^{-1}$ to $100 \mathrm{~s}^{-1}$ ) and at various modemixities, ranging from mode I to equi-mixed mode (loading angles of $0^{\circ}$ to $45^{\circ}$ ). A modified compact mixedmode (CMM) configuration, containing a $500-\mu \mathrm{m}$-thick 
Joints

(a)
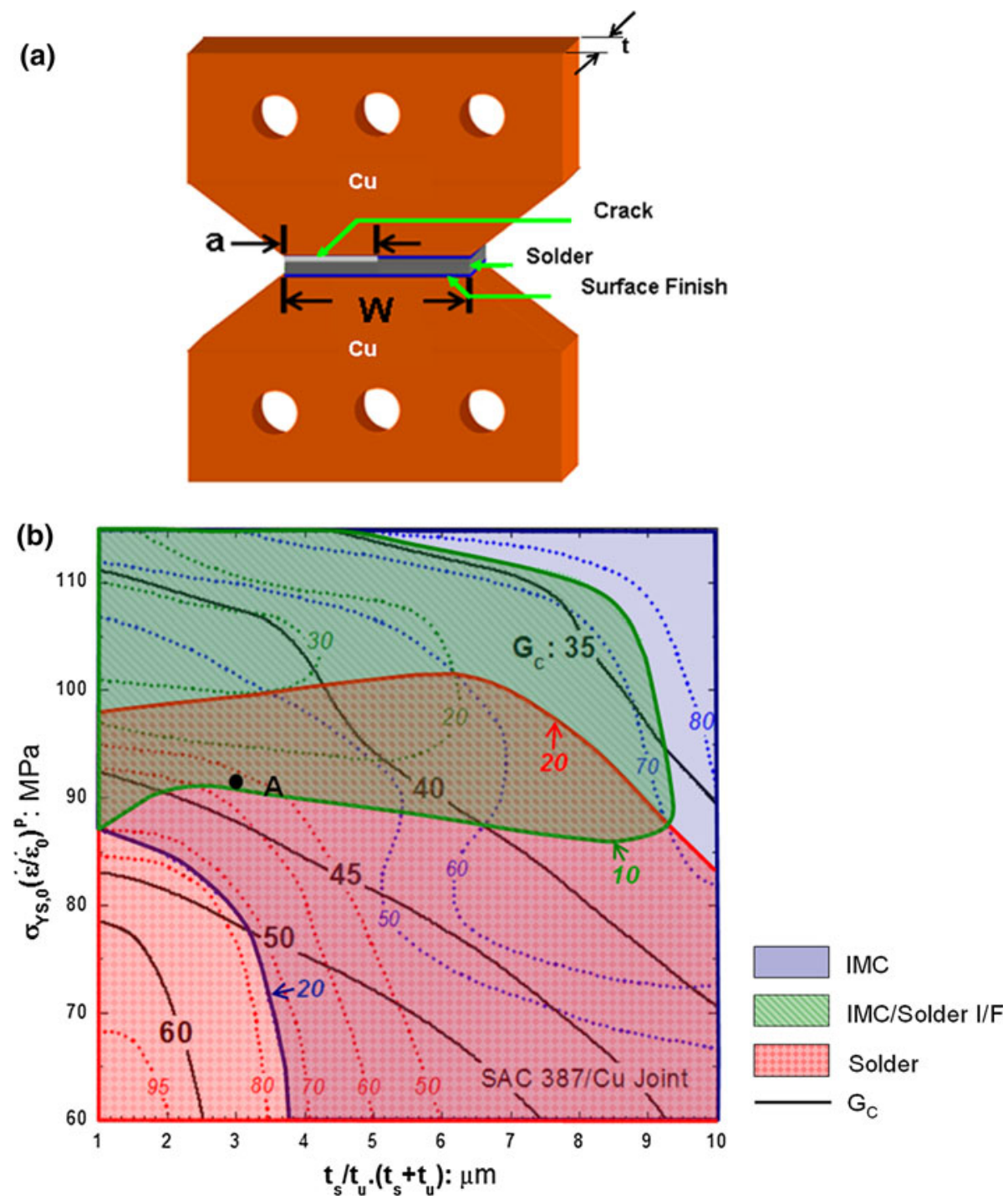

Fig. 1. (a) Schematic of CMM specimen with an interfacial crack. ${ }^{12}$ The interfacial crack was produced by depositing a $500 \mathrm{~nm}$ thick film of Al, which is not wetted by the solder. Details are available in Ref. 12. (b) Mode I fracture mechanism map (FMM) for SAC387/Cu joints. ${ }^{11}$ The colored fields represent different fracture mechanisms, and the color-keyed broken contour lines show the proportion of each fracture type. The solid lines represent iso- $G_{C}$ contours. As an example, for a solder joint with $t_{\text {eff }}=3 \mu \mathrm{m}$ and $\sigma_{\mathrm{ys}, \text { eff }}=92 \mathrm{MPa}$ (represented by point A), $G_{\mathrm{C}} \approx 43 \mathrm{~J} /$ $\mathrm{m}^{2}$, the proportion of solder fracture (red) is $\sim 50 \%$, and the proportion of IMC fracture (blue) is $\sim 40 \%$ (Color figure online).

adhesive solder joint between massive $\mathrm{Cu}$ substrates (Fig. 1a), was used for the experiments. The effect of a range of processing parameters was also studied. The experimental variables studied included: (i) fracture test conditions (strain rate and loading angle), (ii) solder reflow parameters (dwell time and cooling rate), and (iii) postreflow thermal history of the solder joint (aging temperature and time). For each condition, the fracture toughness, expressed as a critical strain energy release rate, $G_{\mathrm{C}}$, was measured, and the fracture surfaces and crack profiles were examined to determine the contributions of various fracture mechanisms (solder fracture, solder-IMC interface fracture, and IMC fracture). The results were finally assembled in the form of a FMM. The main observations of Refs. 10-12 are summarized below.
It was found that, irrespective of the condition, the fracture path was always close to (i.e., associated with) the solder-Cu interfacial region, although the relative contributions of the various fracture mechanisms changed substantially. The fracture toughness, $G_{\mathrm{C}}$, increased as the yield strength of the solder decreased. A low yield strength, and hence a high $G_{\mathrm{C}}$, could be attained by reducing the loading rate, by using a slower cooling rate during reflow, or via postreflow aging of the solder. In general, thicker and rougher (i.e., more scallop-like) IMC layers, which result from longer dwell times during reflow, decreased $G_{\mathrm{C}}$ for a given solder yield strength. For the same IMC thickness, a smoother IMC layer, such as that obtained after aging, increases $G_{\mathrm{C}}$. The lower $G_{\mathrm{C}}$ for thick and rough IMCs is attributable to the 
increased contribution of IMC fracture (and the commensurately lower contribution of solder fracture) to the overall fracture mechanism. It was noted that isothermal aging softened the solder, enhancing fracture toughness, but also resulted in a thicker interfacial IMC layer, which tends to reduce fracture toughness. The final impact of isothermal aging is thus determined by a balance of these opposing factors.

Among the three prevalent mechanisms of joint failure (solder fracture, IMC fracture, or solder-IMC interface fracture), solder and IMC fractures were observed to be the most and the least energy absorbing, respectively. Typically, all three mechanisms operated simultaneously in a given sample, although the relative proportions of their contributions varied, depending on the test conditions and sample history. The key parameters which determined the mix of different fracture mechanisms were the strain-rate-compensated solder yield strength $\left(\sigma_{\mathrm{ys}, \mathrm{eff}}\right)$ and a roughness-compensated IMC thickness $\left(t_{\text {eff }}\right)$. As shown in Fig. 1b, FMMs were plotted in the $\sigma_{\text {ys,eff }}-t_{\text {eff }}$ space, identifying (i) the dominant operating mechanism, (ii) the relative proportions of each mechanism, and (iii) the resultant fracture toughness. The FMMs clearly demonstrated that a combination of hard solder (large $\sigma_{\mathrm{ys}, \text { eff }}$ ) and thick, rough IMCs (large $t_{\text {eff }}$ ) resulted in predominantly IMC fracture and a low $G_{\mathrm{C}}$, whereas a soft solder and a thin, smooth IMC result in predominantly solder fracture and a high $G_{\mathrm{C}}$. These general trends, based on SAC387 joints attached to $\mathrm{Cu}$ substrates, were valid for both mode I and equi-mixed mode loading conditions. ${ }^{11}$

To plot the FMM, the effective (strain-rate-compensated) solder yield strength was expressed as ${ }^{11}$

$$
\sigma_{\mathrm{ys}, \mathrm{eff}}=\sigma_{\mathrm{ys}, \mathrm{o}}\left(\frac{\dot{\varepsilon}}{\dot{\dot{\varepsilon}}_{\mathrm{o}}}\right)^{p}
$$

where $\sigma_{\mathrm{ys}, \mathrm{o}}$ is the solder yield strength at a strain rate of $\dot{\varepsilon}_{o}, \dot{\varepsilon}$ is the strain rate of interest, and $p$ is a material constant. The effective (roughness-compensated) IMC thickness was expressed as ${ }^{11}$

$$
t_{\mathrm{eff}}=\frac{t_{\mathrm{s}}}{t_{\mathrm{u}}}\left(t_{\mathrm{u}}+t_{\mathrm{s}}\right)=t_{\mathrm{s}}+\frac{t_{\mathrm{s}}^{2}}{t_{\mathrm{u}}}
$$

where $t_{\mathrm{s}}, t_{\mathrm{u}}$, and $t_{\mathrm{t}}$ are the IMC scallop height, uniform IMC thickness, and total IMC thickness, respectively, as shown in Fig. 2.

The definition of the effective yield strength used for the FMMs is consistent with a number of reports showing a power-law dependence of solder yield strength on strain rate. ${ }^{13,14}$ The formulation of $t_{\text {eff }}$, on the other hand, was first proposed in Ref. 11, and the functional dependence of the fracture path on $t_{\text {eff }}$ was qualitatively validated based on observations on the solder-Cu system. Inspection of Eq. 2 reveals that $t_{\text {eff }}$ increases with increasing roughness (i.e., the ratio of scallop height $t_{\mathrm{s}}$ to the uniform IMC

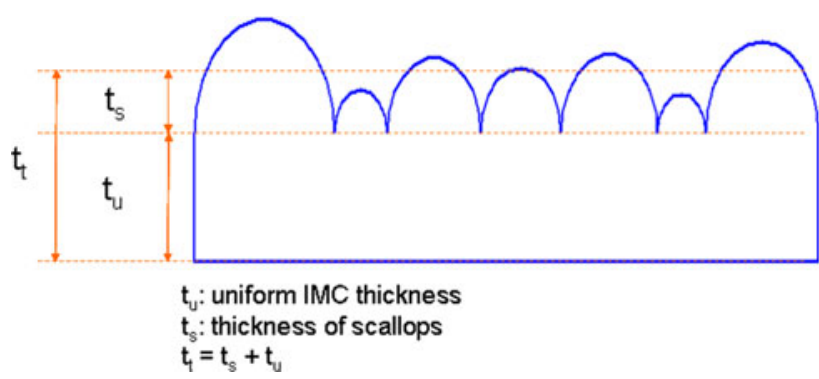

Fig. 2. Typical IMC morphology, and definition of the IMC thickness parameters, $t_{\mathrm{s}}, t_{\mathrm{u}}$, and $t_{\mathrm{t}}{ }^{11}$

thickness $\left.t_{\mathrm{u}}\right)$ and total thickness $\left(t_{\mathrm{u}}+t_{\mathrm{s}}\right)$. However, the particular formulation for $t_{\text {eff }}$ given by Eq. 2 suggests that, when the IMC layer becomes smooth (i.e., when $t_{\mathrm{s}} / t_{\mathrm{u}} \rightarrow 0$ ), $t_{\mathrm{eff}} \rightarrow 0$, even when the layer is very thick. Thus, according to the FMM in Fig. $1 b$, a joint with a very thick IMC layer would result in a very high propensity for solder fracture (low likelihood of IMC fracture), as long as the layer is smooth. In solder-Cu joints, this situation almost never arises, since the IMCs always have significant roughness. However, as shown below, for SAC solder joints on $\mathrm{Ni}$ substrates, the IMCs are often smooth, and yet the failure is through the IMC, as long as it is thick. This necessitates reformulation of the mathematical description of $t_{\text {eff }}$, which is the subject of this paper.

\section{CONSIDERATIONS FOR DEFINING EFFECTIVE IMC THICKNESS}

In developing an expression for $t_{\text {eff }}$, the following two physical characteristics of solder joint fracture are considered: (i) the fracture toughness of solder joints decreases with increasing IMC thickness, since the probability of existence of critical-length cracks in the brittle IMC layer increases with its thickness, and (ii) $G_{\mathrm{C}}$ decreases with increasing roughness of the IMC layer (or height of the IMC scallops), which causes strain concentrations in the solder near the scallop tips (i.e., at the solder-IMC interface) during deformation. ${ }^{4,12,15}$ These strain concentrations arise due to misfit strains induced via elastic-plastic mismatch between the solder and the IMC layer. ${ }^{10,12,13}$ In addition, strain localization within the solder immediately adjacent to and parallel to the interface can cause stress concentrations in the scallops, enhancing the propensity for IMC fracture. ${ }^{10}$ Therefore, a rougher IMC layer forces crack propagation either along the solder-IMC interface via microvoid nucleation and growth or through the IMC layer and/or the IMC scallops; all of these lead to low fracture toughness. ${ }^{12}$ Therefore, the definition of $t_{\text {eff }}$ must incorporate both the thickness and the roughness of the interfacial IMC. The previously used definition of $t_{\text {eff }}$ (Eq. 2) satisfies this criterion. ${ }^{11,12}$

The total IMC thickness $t_{\mathrm{t}}$ in the solder-Cu samples studied previously ${ }^{11,12}$ was less than $5 \mu \mathrm{m}$, 
(a)

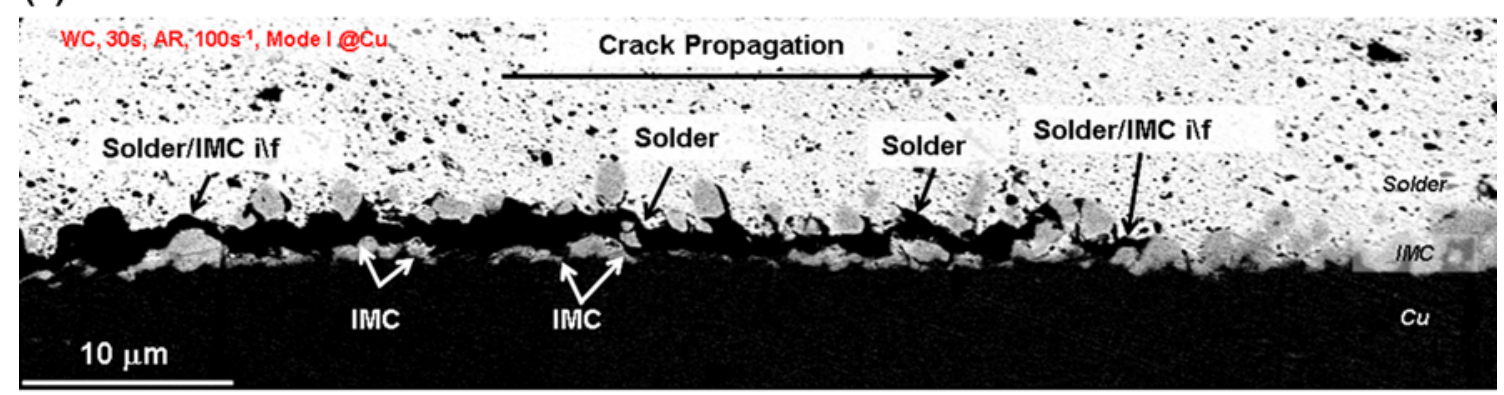

(b)

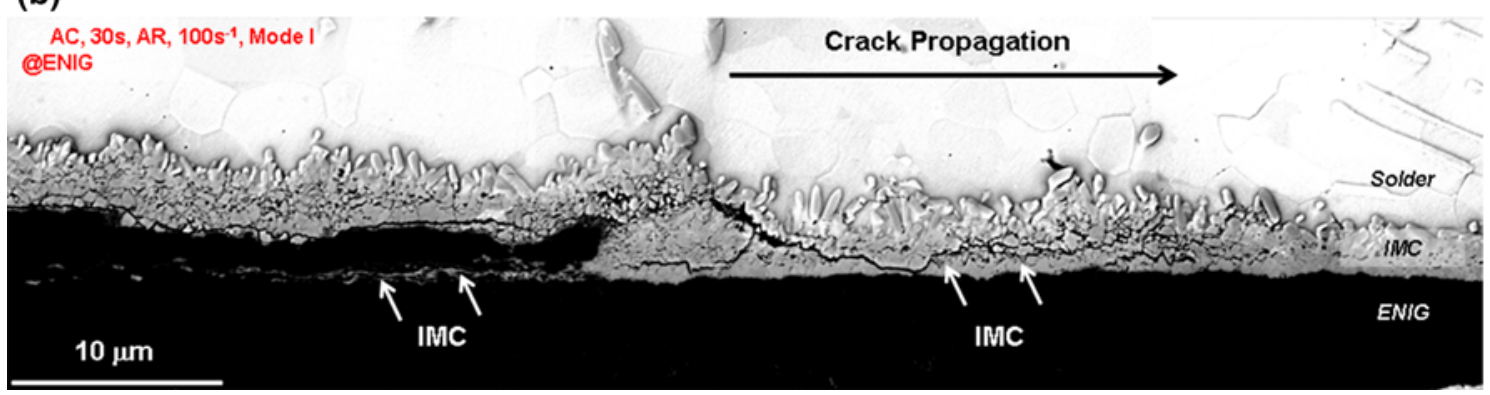

Fig. 3. Crack profiles of (a) WC $30 \mathrm{~s}$ AR Cu substrates, and (b) AC $30 \mathrm{~s}$ AR ENIG substrates. Both samples were tested at strain rate of $100 \mathrm{~s}^{-1}$ and in mode I (WC, water cooled; AC, air cooled; dwell time during soldering, $30 \mathrm{~s}$; AR, as-reflowed).

and $t_{\mathrm{s}} / t_{\mathrm{u}}$ was as large as 2.8. Unlike such "thin and rough" IMC, the IMC layers in solder-Ni joints are usually "smooth and thick," in both severely aged as well as slow-cooled (air- or furnace-cooled) samples, as discussed below.

The interfacial region in joints of SAC387 on Ni-plated $\mathrm{Cu}$ substrates in the same CMM specimen geometry used previously was observed experimentally. The $\mathrm{Ni}$ metallization was produced by coating the $\mathrm{Cu}$ CMM substrates with $5.4 \mu \mathrm{m}(5 \mu \mathrm{m}$ $\mathrm{Ni}+0.4 \mu \mathrm{m} \mathrm{Au})$ thick electroless nickel immersion gold (ENIG). Figure 3 a shows an as-reflowed SAC387/Cu joint (sample A) formed at $260^{\circ} \mathrm{C}$ with a dwell time of $30 \mathrm{~s}$, followed by quenching in water (WC). Figure 3b shows an as-reflowed SAC387/ ENIG joint (sample B) formed at the same temperature with the same dwell time but cooled in air (AC) following soldering. Comparison of the two figures clearly reveals that, whereas the solder-Cu interface is rough and thin, the solder-Ni interface is smooth and thick. Table I presents the corresponding IMC thicknesses $\left(t_{\mathrm{s}}, t_{\mathrm{u}}, t_{\mathrm{t}}\right.$, and $\left.t_{\mathrm{eff}}\right)$ and $\sigma_{\text {ys,eff }}$ values for these two samples. Clearly, the IMC layer in sample $\mathrm{B}$ is thicker (large $t_{\mathrm{t}}$ ) and much smoother (small $t_{\mathrm{s}} / t_{\mathrm{u}}$ ) as compared with sample A. As shown in Table I, this results in a significantly smaller $t_{\text {eff for sample B. }}$.

In addition, $\sigma_{\text {ys,eff }}$ of sample B was much smaller than that of sample A. Since both $t_{\text {eff }}$ (as predicted by Eq. 2) and $\sigma_{\text {ys,eff }}$ for sample B are smaller than those for sample $\mathrm{A}$, the relative contribution of fracture through solder should be larger in sample B. ${ }^{11,12} \mathrm{On}$ the contrary, however, Fig. 3 reveals that the crack in sample $\mathrm{B}$ is more prone to propagate through the IMC layer than in sample A. This discrepancy can be attributed to the definition of $t_{\text {eff }}$ as given by Eq. 2, as discussed above. The inconsistency becomes increasingly egregious as $t_{\mathrm{s}} / t_{\mathrm{u}}$ approaches 0 , whereupon Eq. 2 predicts that the crack would pass through the solder instead of the brittle IMC layer, even when it is very thick (i.e., $t_{\mathrm{u}} \rightarrow \infty$ ). Clearly, Fig. 3b shows that this inference is problematic. This nonphysicality arises mainly due to the fact that $t_{\mathrm{eff}}$, as defined by Eq. 2, has a much stronger dependence on $t_{\mathrm{s}}$ than on $t_{\mathrm{u}}$, * and therefore results in underes-

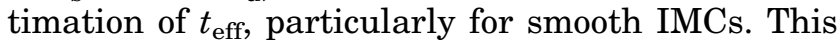
produces reasonable results for the thin-IMC case, when the propensity of the crack to propagate through the uniform part of the IMC layer is minimal, as in the solder-Cu case. However, this becomes problematic when $t_{\mathrm{u}}$ is large (and/or $t_{\mathrm{s}} / t_{\mathrm{u}}$ is small).

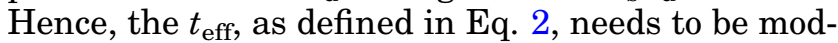
ified to adapt to both "thick and smooth" and "thin and rough" interfacial IMC layers.

\section{MODIFICATION OF THE DEFINITION OF $T_{\text {eff }}$}

Based on the above discussion, it is evident that the definition of $t_{\text {eff }}$ must satisfy the following requirements, in addition to the criteria outlined earlier: First, $t_{\text {eff }}$ should be finite and significant in the two limiting cases corresponding to $t_{\mathrm{s}} \rightarrow 0$ or $t_{\mathrm{u}} \rightarrow 0$ (i.e., very smooth or very thin IMC). Secondly, $t_{\text {eff }}$ should be dominated by $t_{\mathrm{u}}$ when $t_{\mathrm{u}}$ is very

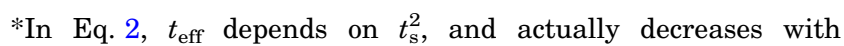
increasing $t_{\mathrm{u}}$. 
Table I. Summary of some of the critical parameters of samples shown in Fig. 3

\begin{tabular}{|c|c|c|c|c|c|c|c|}
\hline$t_{\mathbf{u}}(\mu \mathbf{m})$ & $\boldsymbol{t}_{\mathrm{s}}(\boldsymbol{\mu m})$ & $\boldsymbol{t}_{\mathrm{t}}(\boldsymbol{\mu} \mathbf{m})$ & $t_{\mathrm{u}} / t_{\mathrm{s}}$ & $t_{\text {eff }}$ (Eq. 2) & $t_{\text {eff }}$ (Eq. 3) & $\sigma_{\mathbf{y s}, \text { eff }}(\mathbf{M P a})$ & Solder Mechanism (\%) \\
\hline 0.44 & 0.77 & 1.21 & 0.57 & 2.12 & 1.7 & 105 & 57.07 \\
\hline 4.94 & 1.08 & 6.02 & 4.57 & 1.32 & 6.22 & 74 & 31.73 \\
\hline
\end{tabular}

large relative to $t_{\mathrm{s}}$, and by $t_{\mathrm{s}}$ when $t_{\mathrm{s}} \gg t_{\mathrm{u}}$. Based on these considerations, a modified expression for $t_{\text {eff }}$ is proposed, where the term $t_{\mathrm{u}}$ in Eq. 2 has been replaced by the total thickness $t_{\mathrm{t}}$, as follows:

$$
t_{\mathrm{eff}}=t_{\mathrm{t}}+\frac{t_{\mathrm{s}}^{2}}{t_{\mathrm{t}}}=t_{\mathrm{u}}+t_{\mathrm{s}}+\frac{t_{\mathrm{s}}^{2}}{t_{\mathrm{u}}+t_{\mathrm{s}}} .
$$

This modified definition of $t_{\text {eff }}$ satisfies all of the criteria set out above; For instance, when $t_{\mathrm{s}}=0$ and $t_{\mathrm{u}} \neq 0$, then $t_{\text {eff }}$ is determined by $t_{\mathrm{u}}\left(t_{\mathrm{eff}}=t_{\mathrm{u}}\right)$. On the other hand, when $t_{\mathrm{u}}=0$ but $t_{\mathrm{s}} \neq 0$, then $t_{\mathrm{eff}}$ is dependent only on $t_{\mathrm{s}}\left(t_{\text {eff }}=2 t_{\mathrm{s}}\right)$. It is further noted that the effects of all these limiting conditions on the joint fracture behavior are consistent with the experimental data on both $\mathrm{Cu}$ and $\mathrm{Ni}$ substrates obtained to date. In the following, the efficacy of the modified $t_{\text {eff }}$ in capturing the effects of $t_{\mathrm{t}}, t_{\mathrm{u}}$, and $t_{\mathrm{s}}$ on the fracture behavior of solder joints is discussed.

\section{Case 1: Total Thickness $\left(t_{t}\right)$ Varies While Roughness $\left(t_{\mathrm{s}}\right)$ Remains Constant}

For constant $t_{\mathrm{s}}$, the effect of variation of $t_{\mathrm{u}}$ on $t_{\mathrm{eff}}$ may be written as follows:

$$
\frac{\partial t_{\mathrm{eff}}}{\partial t_{\mathrm{u}}}=1-\frac{t_{\mathrm{s}}^{2}}{\left(t_{\mathrm{u}}+t_{\mathrm{s}}\right)^{2}} .
$$

Since $t_{\mathrm{s}} \leq t_{\mathrm{u}}+t_{\mathrm{s}}$, or $\partial t_{\mathrm{eff}} / \partial t_{\mathrm{u}} \geq 0$, therefore $t_{\mathrm{eff}}$ will increase with $t_{\mathrm{u}}$. This implies that an increase in $t_{\mathrm{u}}$ while keeping $t_{\mathrm{s}}$ constant should adversely affect the fracture toughness $\left(G_{\mathrm{C}}\right)$ of solder joints. This is consistent with the experimental data presented in Fig. 4a, which shows that severe aging did not necessarily change the roughness $\left(t_{\mathrm{s}}\right)$ of the IMC layer, but increased $t_{\mathrm{u}}$ by $\sim 130 \%$, leading to a net increase of $88 \%$ in $t_{\text {eff. }}$ Despite the fact that severe aging reduces the yield strength of a solder, ${ }^{10-12}$ this large increase in $t_{\text {eff }}$ led to a net decrease in the fracture toughness value by more than $50 \%$.

\section{Case 2: Total Thickness $\left(t_{t}\right)$ Varies While Uniform Thickness $\left(t_{u}\right)$ Remains Constant}

If $t_{\mathrm{u}}$ is kept constant, the variation in $t_{\mathrm{eff}}$ with $t_{\mathrm{s}}$ can be determined as follows:

$$
\frac{\partial t_{\mathrm{eff}}}{\partial t_{\mathrm{s}}}=2-\frac{t_{\mathrm{u}}^{2}}{\left(t_{\mathrm{u}}+t_{\mathrm{s}}\right)^{2}} .
$$

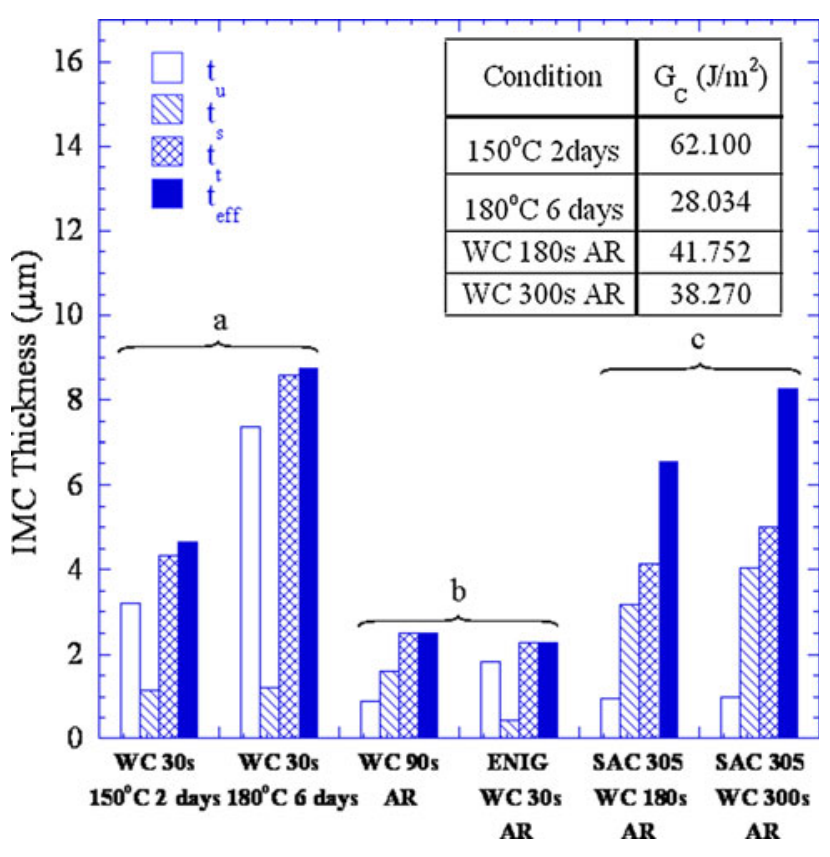

Fig. 4. Variation in $t_{\mathrm{eff}}$ and $G_{\mathrm{C}}$ as a result of: (a) variation in $t_{\mathrm{u}}$ while keeping $t_{\mathrm{s}}$ constant, (b) variation in $t_{\mathrm{u}}$ while keeping $t_{\mathrm{t}}$ constant, and (c) variation in $t_{\mathrm{s}}$ while keeping $t_{\mathrm{u}}$ constant. The solder used is SAC387 and the substrate is pure $\mathrm{Cu}$, unless otherwise specified.

Since $t_{\mathrm{u}} \leq t_{\mathrm{u}}+t_{\mathrm{s}}$, or $\partial t_{\mathrm{eff}} / \partial t_{\mathrm{s}} \geq 0, t_{\mathrm{eff}}$ will increase with $t_{\mathrm{s}}$ and hence deteriorate the fracture toughness of a solder joint. This is also consistent with the experimental observations. As shown in Fig. $4 \mathrm{c}, t_{\mathrm{s}}$ increased by $27 \%$ upon increasing the dwell time during soldering from $180 \mathrm{~s}$ to $300 \mathrm{~s}$, but $t_{\mathrm{u}}$ remained similar (only increasing by $3 \%$ ). This led to a net increase of $26 \%$ in the value of $t_{\text {eff. }}$ This finally resulted in a $\sim 10 \%$ decrease in the $G_{\mathrm{C}}$ value. Considering that the solder yield strength is insensitive to the dwell time during soldering, ${ }^{11}$ this decrease in $G_{\mathrm{C}}$ can be attributed to the increased $t_{\text {eff. }}$

\section{Case 3: Total Thickness $\left(t_{t}\right)$ Remains Constant While Roughness $\left(t_{\mathrm{s}}\right)$ Changes}

If $t_{\mathrm{t}}=t_{\mathrm{s}}+t_{\mathrm{u}}$ is kept constant, and $t_{\mathrm{u}}$ as well as $t_{\mathrm{s}}$ are varied, then their effects on $t_{\text {eff }}$ can be determined as

$$
\frac{\partial t_{\mathrm{eff}}}{\partial t_{\mathrm{s}}}=\frac{2 t_{\mathrm{s}}}{t_{\mathrm{t}}}
$$


(a)

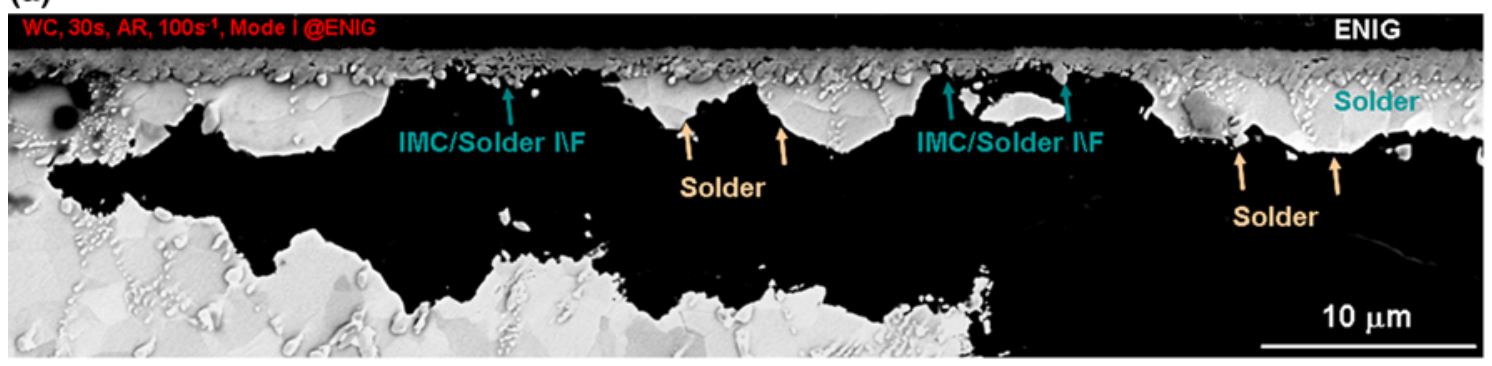

(b)

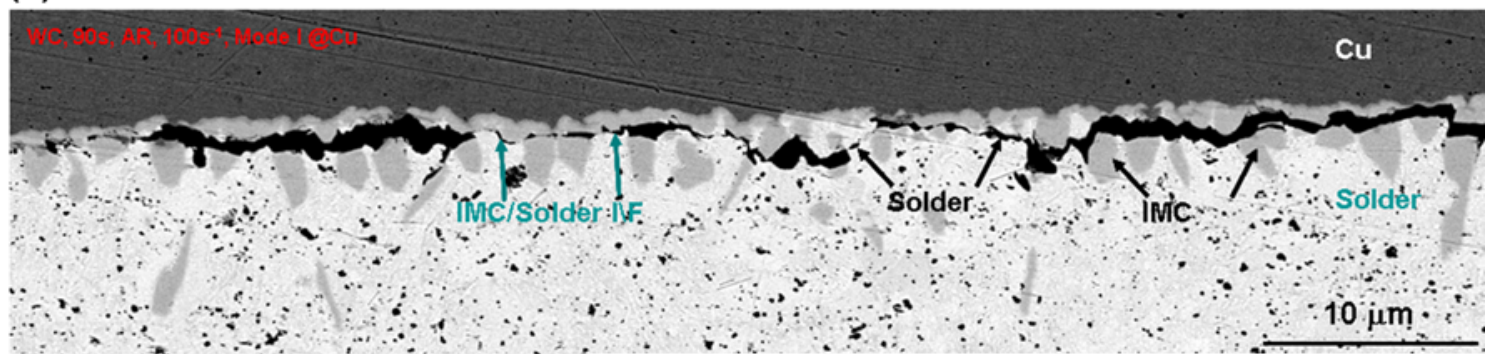

Fig. 5. Crack profiles of (a) WC $30 \mathrm{~s}$ AR ENIG substrates, and (b) WC $90 \mathrm{~s}$ AR Cu substrates. Both samples were tested at strain rate of $100 \mathrm{~s}^{-1}$ and in mode I (WC, water cooled; dwell time during soldering, $30 \mathrm{~s}$ or $90 \mathrm{~s}$; AR, as-reflowed).

$$
\frac{\partial t_{\mathrm{eff}}}{\partial t_{\mathrm{u}}}=\frac{2\left(t_{\mathrm{u}}-t_{\mathrm{t}}\right)}{t_{\mathrm{t}}}
$$

Since $0 \leq t_{\mathrm{s}}, t_{\mathrm{u}} \leq t_{\mathrm{t}}, \partial t_{\mathrm{eff}} / \partial t_{\mathrm{s}} \geq 0$, and $\partial t_{\mathrm{eff}} / \partial t_{\mathrm{u}} \leq 0$. Therefore, $t_{\mathrm{eff}}$ would increase if either $t_{\mathrm{s}}\left(\right.$ or $\left.t_{\mathrm{s}} / t_{\mathrm{u}}\right)$ is increased, or $t_{\mathrm{u}}$ is decreased while keeping $t_{\mathrm{t}}$ constant. This suggests that planar IMC layers perform better than a nodular or scallop-like IMC layer in improving the fracture toughness of the solder joints. Again, this is consistent with experimental results. As shown in Fig. 4 b, $t_{\mathrm{t}}$ for a SAC387/Cu joint (as-reflowed, dwell time $=90 \mathrm{~s}$, water cooled) was similar to that for a SAC387/ENIG joint (as-reflowed, dwell time $=30 \mathrm{~s}$, water cooled), showing only a $9.6 \%$ difference. However, the ratio $t_{\mathrm{s}} / t_{\mathrm{u}}$ in the former sample was $~ 84 \%$ larger than that in the latter, indicating that the IMC in the SAC-ENIG joint is much smoother than in the SAC-Cu joint.

IMC morphological differences in solder joints with different metallizations cannot be directly correlated with differences in $G_{\mathrm{C}}$.** Still, it is likely that the effect of IMC morphology on the joint fracture mechanism is similar for different metallizations (since the large difference between solder and IMC properties in both systems renders modest differences in IMC properties relatively inconsequential from the point of view of deformation or fracture mechanisms). Therefore, the effect of IMC roughness may be inferred by comparing solder

\footnotetext{
**This is because differences in IMC composition, elastic properties, and fracture toughness, as well as differences in solderIMC interfacial properties, are likely to have significantly different effects on $G_{\mathrm{C}}$ in different systems.
}

joints on ENIG and $\mathrm{Cu}$ of similar IMC uniform thickness but different roughness. Figure 5 shows the crack profiles in solder-ENIG and solder-Cu joints with similar $t_{\mathrm{u}}$ values, but with the Ni IMC having a much smaller $t_{\mathrm{s}}$ than the $\mathrm{Cu}$ IMC. It is seen that the crack is more prone to propagate through the ductile solder in the solder-Ni joint, which has a smooth IMC ( small $t_{\mathrm{s}}$ ), than in the solder-Cu joint with a rough IMC layer. As Eq. 3 suggests, the smoother IMC produces a smaller $t_{\text {eff }}$, and since higher $G_{\mathrm{C}}$ values are associated with solder fracture (as opposed to IMC fracture), a smoother IMC will tend to enhance the fracture toughness of the solder joint.

\section{FRACTURE MECHANISM MAP WITH MODIFIED $\boldsymbol{T}_{\text {eff }}$}

In Fig. 6a, the FMM for SAC387/Cu joints shown in Fig. $1 \mathrm{~b}$ is replotted using the modified definition of $t_{\text {eff }}$ given by Eq. 3 . The scale of $t_{\text {eff }}$ is somewhat compressed in the updated map, but the trends for both $G_{\mathrm{C}}$ and the fracture mechanism (as indicated by the associated contour lines) remain similar to the map using the previous definition of $t_{\text {eff }}$. Consistent with the FMM in Fig. 1b, the new FMM in Fig. $6 \mathrm{a}$ also indicates that a combination of low $\sigma_{\mathrm{ys}, \text { eff }}$ and low $t_{\text {eff }}$ results in a tougher solder joint. To validate the effectiveness of the updated definition of $t_{\text {eff }}$ even for joints containing a thick and smooth IMC layer, a few samples were aged at $180^{\circ} \mathrm{C}$ for $144 \mathrm{~h}$. This resulted in a solder joint with $t_{\mathrm{t}}$ of $\sim 8.6 \mu \mathrm{m}$ and $t_{\mathrm{s}} / t_{\mathrm{u}}$ of $\sim 0.16$ and hence $t_{\mathrm{eff}}$, as calculated using Eq. 3, was equal to $8.8 \mu \mathrm{m}$. Following the procedure outlined in Ref.11, the effective yield strength of the solder $\left(\sigma_{\mathrm{ys}, \mathrm{eff}}\right)$ at strain rate of 
(a)

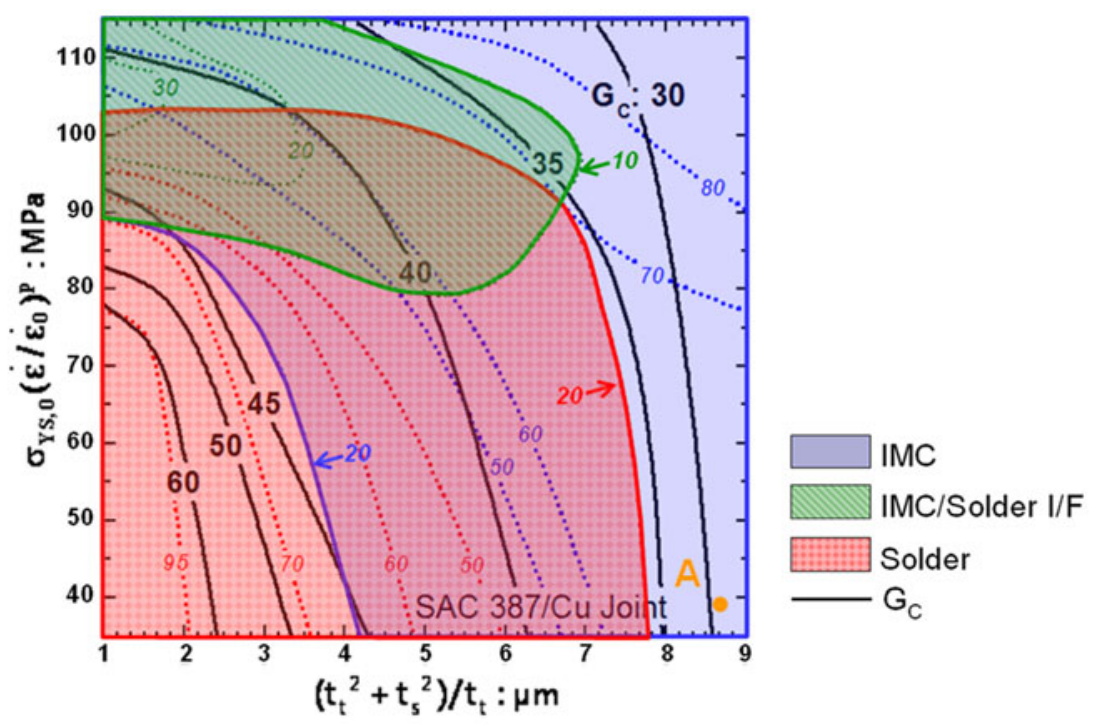

(b)

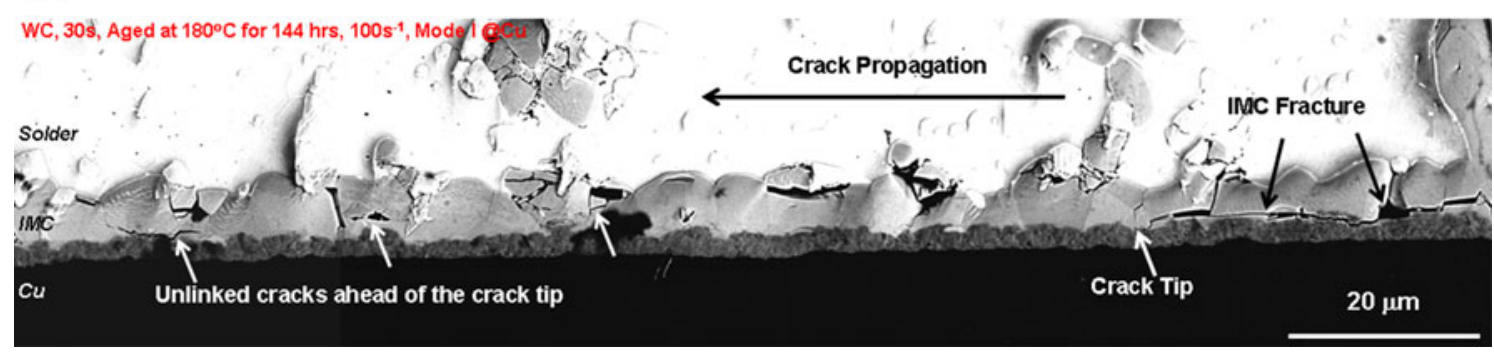

Fig. 6. (a) Updated mode I fracture mechanism map for SAC387/Cu joints using $t_{\text {eff }}=t_{\mathrm{t}}+t_{\mathrm{s}} \cdot t_{\mathrm{s}} / t_{\mathrm{t}}$ as $x$-axis. (b) Crack profile of SAC387/Cu joints processed with WC, $30 \mathrm{~s}$, aged for $144 \mathrm{~h}$ at $180^{\circ} \mathrm{C}$. The sample was tested at $100 \mathrm{~s}^{-1}$ strain rate under mode I loading (Color figure online).

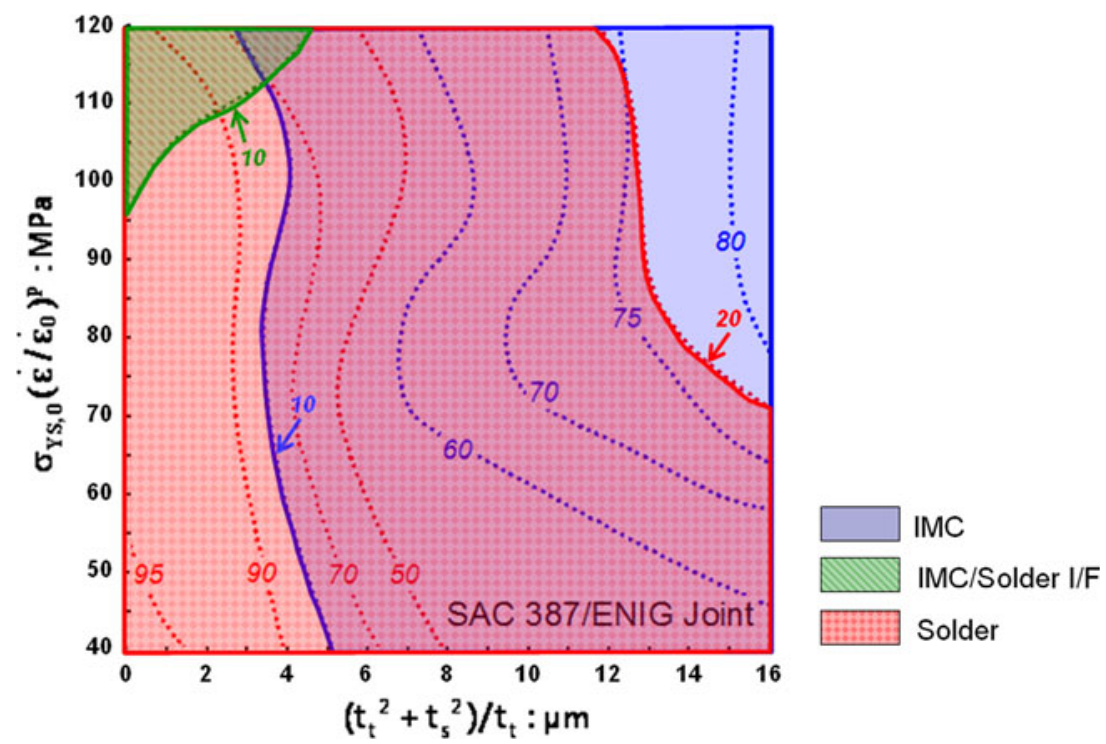

Fig. 7. Preliminary mode I fracture mechanism map for SAC387/ENIG joints, showing only the mechanisms and fracture proportions $\left(G_{\mathrm{C}}\right.$ data not shown) (Color figure online).

$100 \mathrm{~s}^{-1}$ was determined to be equal to $39.1 \mathrm{MPa}$. Point A on Fig. 6a represents this solder joint, for which the FMM predicts a $G_{\mathrm{C}}$ value of slightly less than $30 \mathrm{~J} / \mathrm{m}^{2}$, and crack propagation primarily through the interfacial IMC layer. Consistent with the modified FMM, the experimentally measured 
value of $G_{\mathrm{C}}$ for this joint was $28 \mathrm{~J} / \mathrm{m}^{2}$. Furthermore, as shown in Fig. $6 \mathrm{~b}$, the crack, as well as the damage zone ahead of the crack tip, remained predominantly in the IMC layer in this sample, as expected from the FMM. This validates the modification proposed for the definition of $t_{\text {eff }}$ as given by Eq. 3, and suggests that it is valid for both smooth, thick IMCs, as well as rough, thin IMCs.

Figure 7 shows a preliminary mode I FMM for SAC387/ENIG joints. This was developed using the same methodology as that for the SAC387/Cu joints. Compared with the mode I FMM for SAC387/Cu joints, the span of the solder fracture field becomes more extensive for SAC387/ENIG joints. This is consistent with the above discussion (and Fig. 5a), which showed that the smoother IMC in solder-Ni joints promotes solder fracture and reduces the contribution of IMC fracture; For example, for $\sigma_{\mathrm{ys}, \text { eff }}=80 \mathrm{MPa}$, the $t_{\text {eff }}$ required for $20 \%$ solder fracture in the SAC387/ENIG joint is almost twice as much as that in SAC387/Cu joints $(14 \mu \mathrm{m}$ versus $7.2 \mu \mathrm{m})$. Since the IMC in the solder-ENIG joint is smoother than that in solder-Cu joints, this suggests that a much thicker IMC is needed in the solder-Ni joint to have the same mix of fracture mechanisms as in a solder-Cu joint. In other words, this indicates that, because of the lower $t_{\text {eff }}$ in SAC387/ENIG joints, the mechanism of IMC fracture in these joints is suppressed relative to the SAC387/Cu joints.

\section{CONCLUSIONS}

The morphology of the interfacial IMC layer in solder joints has been characterized in terms of an effective thickness, $t_{\text {eff }}$. A previously used definition of $t_{\text {eff }}^{11}$ was modified to make it consistent with physical mechanistic trends observed in both solder-Cu and solder-Ni joints. The new definition of $t_{\text {eff }}$ resolves discrepancies noted for smooth interfaces with the previously used definition of $t_{\text {eff }}$, by ensuring proper emphasis of the total thickness of the IMC layer, and makes it compatible with both thin, rough IMC layers as well as thick, smooth IMC layers. FMMs were plotted using this new definition of $t_{\text {eff }}$ for both solder-Cu and solder-Ni systems. The mechanistic trends of FMMs plotted using both the previous and new definitions of $t_{\text {eff }}$ were found to be self-consistent, although the precise locations of the field boundaries were slightly different. The validity of the new definition was verified by comparing the predictions of the resulting FMMs with experimental observations of the crack path for several different IMC morphologies and thicknesses in the two systems. Although the new formulation for $t_{\text {eff }}$ is developed for solder joints, the generalized approach presented is expected to be valid for a wide range of joints with interfacial interphase layers.

\section{ACKNOWLEDGEMENTS}

This research was supported by NSF Grant No. DMR-0939392, SRC Task Nos. 1855 and 1292.088, and SERDP Contract No. W912HQ-10-C-0041.

\section{REFERENCES}

1. B. Zhang, H. Ding, and X. Sheng, Microelectron. Reliab. 49, 530 (2009).

2. J.G. Lee, A. Telang, K.N. Subramanian, and T.R. Bieler, J. Electron. Mater. 31, 1152 (2002).

3. J.H.L. Pang, B.S. Xiong, and T.H. Low, 54th Electronic Components and Technology Conference, Las Vegas, 2004.

4. S.M. Hayes, N. Chawla, and D.R. Frear, Microelectron. Reliab. 49, 269 (2009).

5. K.S. Siow and M. Manoharan, Mater. Sci. Eng. A 404A, 244 (2005).

6. S.M. Joo and H.K. Kim, Mater. Sci. Eng. A 528, 2711 (2011).

7. X. Li, F. Li, F. Guo, and Y. Shi, J. Electron. Mater. 40, 51 (2011)

8. S.V. Nadimpalli and J.K. Spelt, Mater. Sci. Eng. A 527, 724 (2010).

9. D.S. Liu, C.Y. Kuo, C.L. Hsu, G.S. Shen, Y.R. Chen, and K.C. Lo, Mater. Sci. Eng. A 494, 196 (2008).

10. P. Kumar, I. Dutta, V. Sarihan, D.R. Frear, and M. Renavikar, Proc. 11th Intersociety Conf. on Thermal and Thermomechanical Phenomena in Electronic Systems (ITherm) (Orlando: IEEE/ASME, 2008).

11. P. Kumar, Z. Huang, I. Dutta, R. Sidhu, M. Renavikar, and R. Mahajan, J. Electron. Mater. 41, 412 (2012).

12. Z. Huang, P. Kumar, I. Dutta, J.H.L. Pang, R. Sidhu, M. Renavikar, and R. Mahajan, J. Electron. Mater. 41, 375 (2012).

13. X. Long, R. Guduru, I. Dutta, V. Sarihan, and D.R. Frear, J. Electron. Mater. 37, 189 (2008).

14. J.H.L. Pang and B.S. Xiong, IEEE Trans. Compon. Packag. Technol. 28, 830 (2005).

15. X. Deng, R.S. Sidhu, P. Johnson, and N. Chawla, Metall. Mater. Trans. 36A, 55 (2005). 